\title{
Frailty and Co-Prescribing of Potentially Interacting Drugs in New Users of Warfarin
}

\author{
Milka Hauta-aho ${ }^{1,2,3}$ (D) Simo Teperi ${ }^{4} \cdot$ Maarit J. Korhonen $^{1,5} \cdot \mathrm{J}_{\text {. Simon Bell }}^{5,6} \cdot$ Nicholas Farinola $^{3}$ (1) $\cdot$ Sally Johns ${ }^{3}$. \\ Sepehr Shakib ${ }^{3,7}$ (D) Risto Huupponen ${ }^{1,2}$
}

Published online: 9 March 2020

(c) The Author(s) 2020

\begin{abstract}
Background Warfarin is underutilised in frail older people because of the fear of bleeding complications. Drug interactions are an independent bleeding risk factor. However, the extent to which potential drug interactions are taken into account at warfarin therapy initiation in frail patients is not known.

Objective The objective of this study was to investigate the use of potentially interacting drugs increasing the bleeding risk before and after warfarin initiation in frail and non-frail patients.

Methods We conducted an observational study including inpatients aged $\geq 60$ years initiated on warfarin in a tertiary hospital in Adelaide, South Australia. Frailty status was assessed with the Reported Edmonton Frail Scale. Medication charts were reviewed before and after warfarin initiation.

Results In total, 151 patients (102 non-frail and 49 frail) were included. Before warfarin initiation, the use of clopidogrel and acetaminophen was more common in frail patients compared with non-frail patients $(25.5 \%$ vs $10.2 \%, p=0.0135,63.8 \%$ vs $35.7 \% p=0.0014$, respectively). The use of non-steroidal anti-inflammatory drugs, $9.2 \%$ in non-frail patients and $6.4 \%$ in frail patients before warfarin initiation, was completely stopped after warfarin initiation in both groups. The use of antiplatelet drugs decreased from $56.1 \%$ in non-frail patients and $66.0 \%$ in frail patients to $12.2 \%$ and $14.9 \%$, respectively. Instead, the use of drugs affecting the metabolism of warfarin or vitamin $\mathrm{K}$ increased in both groups. No statistically significant difference was seen in the exposure to interacting drugs between study groups after warfarin initiation. Acetaminophen, senna glycosides and cytochrome P450 2C9 inhibiting drugs were the most common interacting drugs at discharge used in $49.0 \%$, $18.4 \%$ and $20.4 \%$ of non-frail patients and $53.2 \%, 29.8 \%$ and $19.1 \%$ of frail patients, respectively.

Conclusions The overall frequency of potential drug interactions was moderate and frail patients were not exposed to warfarin drug interactions more often than non-frail patients. Further studies in larger study populations are required to verify these results.
\end{abstract}

\section{Introduction}

Bleeding is a common and potentially serious complication of warfarin therapy. The risk is increased with advanced age $[1,2]$ and drug interactions $[3,4]$. The fear of serious bleeding complications is the key reason for the underutilisation of warfarin, especially in frail older people, as shown by previous studies $[5,6]$. Considering the high prevalence of

Electronic supplementary material The online version of this article (https://doi.org/10.1007/s40266-020-00755-0) contains supplementary material, which is available to authorized users.

Milka Hauta-aho

milka.hauta-aho@utu.fi

Extended author information available on the last page of the article comorbidities and polypharmacy in frail patients [7], the risk of drug interactions is high in this population.

Frail patients are more vulnerable to adverse drug reactions [8], and thus the bleeding risk caused by drug interactions may be even higher than reported in non-frail patients. More importantly, the excess risk caused by drug interactions is predictable and often avoidable by choosing alternative medications. Despite this, previous studies show that up to $60-80 \%$ of warfarin users, the majority of whom are older people, are exposed to potential drug interactions [9-12]. However, to our knowledge, there is no existing research on the frequency of warfarin drug interactions in frail patients compared to non-frail patients. Our aim was to examine the exposure to drug interactions increasing the bleeding risk and the number of all regular medications before and after 


\section{Key Points}

The frequency of warfarin drug interactions was moderate and did not differ in frail and non-frail patients after warfarin initiation.

The use of non-steroidal anti-inflammatory drugs and antiplatelets decreased following warfarin initiation in both frail and non-frail patients. Acetaminophen, cytochrome P450 2C9 inhibiting drugs and senna glycosides were the most common interacting drugs in both groups.

Overall, potential drug interactions appeared to be taken into account at warfarin initiation independent of a patient's frailty status.

warfarin initiation with a special focus on frailty status at the early phase of warfarin therapy.

\section{Methods}

\subsection{Study Design, Setting and Subjects}

This observational study was performed at the Royal Adelaide Hospital, in Adelaide, South Australia between October 2012 and May 2013. The study was approved by the institutional human research ethics committee (Royal Adelaide Hospital Protocol No. 120911).

We included previously warfarin-naïve inpatients, aged $\geq 60$ years, in whom warfarin was initiated within 2 weeks for any indication. Eligible patients were identified either by ward pharmacists or from daily International Normalized Ratio (INR) measurement reports provided by the hospital laboratory. Patients were recruited from all units of the hospital. The majority of the patients included in the study were treated in the units of general medicine, cardiothoracic surgery, cardiology and neurology. All participants provided written informed consent to participate. For patients with cognitive impairment, a third-party consent was obtained from patients' next of kin, carer or significant other. We excluded patients with previous warfarin use or severe hearing impairment.

\subsection{Data Collection}

We collected data on patients' clinical characteristics (age, sex, comorbidities, standard haematological, hepatic and renal laboratory parameters, indication for warfarin therapy and reason for hospitalisation) and medication by reviewing patient files and medication charts and by interviewing patients.

\subsection{Frailty Assessment}

Frailty status was assessed at the time of the recruitment by performing the reported version of the Edmonton Frail Scale as modified by Hilmer et al. [13] and utilised in several previous studies [5, 14, 15]. The Edmonton Frail Scale assesses cognition, general health status, functional independence, social support, medication use, nutrition, mood, continence and functional performance [16]. It has been validated for the assessment of frailty by non-medically trained researchers [16]. In the reported version, the observed 'get up and go' is substituted with a self-report of function 2 weeks prior to admission to avoid confounding by the effects of acute illness [13]. The reported Edmonton Frail Scale scores defines patients' frailty status as follows: 0-5 not frail, 6-7 apparently vulnerable, 8-9 mild frailty, 10-11 moderately frail and $12-18$ severe frailty. In the analysis, the frailty scale was collapsed into two categories because of the low number of patients in the categories of the highest frailty. Patients with score of $\geq 8$ were considered frail. Similar merging of categories has been reported in previous studies [5, 13-15].

\subsection{Medication Data}

We collected information on medications used as outpatients prior to the current admission (home medication) and at discharge. Home medication data were obtained by the ward pharmacist as the best available medication history by interviewing the patient, as well as the general practitioner and community pharmacist, where necessary. In the case where a patient had been admitted earlier than 1 week prior to the day of warfarin initiation, home medication was recorded as the medication on the ward 7 days prior to the day of warfarin initiation. Discharge medication data were obtained from discharge summaries in the medical records, also reconciled by the hospital pharmacist.

We collected information on the use of all regular, systemically administered medications. We excluded medication formulations for topical treatment (e.g. topical creams, oral gels, intravaginal preparations) because the extent of systemic absorption is either minimal or uncertain.

\subsection{Interacting Drugs}

We utilised the INXBASE $^{\circledR}$ (previously SFINX ${ }^{\circledR}$ ) drug-drug interaction database [17] in defining warfarin interaction drugs. Based on the INXBASE ${ }^{\circledR}$ search, we chose to study drugs classified as 'to be avoided' and 'special consideration required' with warfarin. We reviewed the use of interacting 
Table 1 Bleeding and stroke risk scores and comorbidity score calculated for each patient

\begin{tabular}{|c|c|}
\hline Risk score and scoring components & Score \\
\hline \multicolumn{2}{|l|}{ Outpatient Bleeding Risk Index (OBRI) [20] } \\
\hline Age $\geq 65$ years & 1 \\
\hline History of stroke & 1 \\
\hline History of gastrointestinal bleeding & 1 \\
\hline $\begin{array}{l}\text { Recent myocardial infarction, haematocrit }<30 \% \text {, } \\
\text { creatinine }>1.5 \mathrm{mg} / \mathrm{dL} \text { or diabetes mellitus }\end{array}$ & 1 \\
\hline Low risk 0 , intermediate risk $1-2$, high risk 3-4 & Maximum 4 \\
\hline \multicolumn{2}{|l|}{ Anticoagulation and risk factors (ATRIA) [21] } \\
\hline Anaemia & 3 \\
\hline Renal disease & 3 \\
\hline Age $\geq 75$ years & 2 \\
\hline Prior bleeding & 1 \\
\hline Hypertension & 1 \\
\hline Low risk $0-3$, intermediate risk 4 , high risk 5-10 & Maximum 10 \\
\hline \multicolumn{2}{|l|}{ HEMORR2HAGES [22] } \\
\hline Hepatic or renal disease & 1 \\
\hline Ethanol abuse & 1 \\
\hline Malignancy & 1 \\
\hline Older age (age $>75$ years) & 1 \\
\hline Reduced platelet count or function & 1 \\
\hline Hypertension (uncontrolled) & 1 \\
\hline Anaemia & 1 \\
\hline Genetic factors & 1 \\
\hline Excessive falls risk & 1 \\
\hline Stroke & 1 \\
\hline Prior bleed & 2 \\
\hline Low risk $0-3$, intermediate risk 4 , high risk 5-10 & Maximum 11 \\
\hline \multicolumn{2}{|l|}{ HAS-BLED $[23,24]$} \\
\hline Hypertension & 1 \\
\hline Abnormal renal function & 1 \\
\hline Abnormal liver function & 1 \\
\hline Stroke & 1 \\
\hline Bleeding history or predisposition & 1 \\
\hline Labile INR & 1 \\
\hline Elderly & 1 \\
\hline Drugs concomitantly & 1 \\
\hline Alcohol concomitantly & 1 \\
\hline Low risk $0-1$, intermediate risk $2-3$, high risk $\geq 4$ & Maximum 9 \\
\hline \multicolumn{2}{|l|}{$\mathrm{CHADS}_{2}[25]$} \\
\hline Congestive heart failure & 1 \\
\hline Hypertension & 1 \\
\hline Age $\geq 75$ years & 1 \\
\hline Diabetes mellitus & 1 \\
\hline Stroke & 2 \\
\hline Low risk 0 , intermediate risk $1-2$, high risk $\geq 3$ & Maximum 6 \\
\hline \multicolumn{2}{|l|}{$\mathrm{CHA}_{2} \mathrm{DS}_{2}$-Vasc [26] } \\
\hline Congestive heart failure & 1 \\
\hline Hypertension & 1 \\
\hline Age $\geq 65$ years & 1 \\
\hline
\end{tabular}

Table 1 (continued)

\begin{tabular}{|c|c|}
\hline Risk score and scoring components & Score \\
\hline Age $\geq 75$ years & 2 \\
\hline Diabetes mellitus & 1 \\
\hline Stroke & 2 \\
\hline Vascular disease & 1 \\
\hline Female & 1 \\
\hline Low risk 0 , intermediate risk 1 , high risk $\geq 2$ & Maximum 9 \\
\hline \multicolumn{2}{|l|}{ Charlson Comorbidity Index [18] } \\
\hline Myocardial infarct & 1 \\
\hline Congestive heart failure & 1 \\
\hline Peripheral vascular disease & 1 \\
\hline Cerebrovascular disease & 1 \\
\hline Dementia & 1 \\
\hline Chronic pulmonary disease & 1 \\
\hline Connective tissue disease & 1 \\
\hline Ulcer disease & 1 \\
\hline Mild liver disease & 1 \\
\hline Diabetes mellitus & 1 \\
\hline Hemiplegia & 2 \\
\hline Moderate or severe renal disease & 2 \\
\hline Diabetes mellitus with end organ damage & 2 \\
\hline Any tumour & 2 \\
\hline Leukaemia & 2 \\
\hline Lymphoma & 2 \\
\hline Moderate or severe liver disease & 3 \\
\hline Metastatic solid tumour & 6 \\
\hline AIDS & 6 \\
\hline
\end{tabular}

AIDS Acquired Immune Deficiency Syndrome, INR International Normalized Ratio

drugs prior to warfarin initiation and at discharge. The list of these interacting drugs is provided in File 1 of the Electronic Supplementary Material.

\subsection{Risk Scores}

We calculated bleeding and stroke risk scores and the comorbidity index [18] for each patient (Table 1). The HASBLED score was calculated without scoring 'Labile INR values' and HEMORR2HAGES without information on the cytochrome $\mathrm{P} 450$ (CYP) 2C9 genotype as reported by previous articles $[6,19]$.

\subsection{Statistical Analysis}

Relationships between dependent and independent variables were studied with descriptive statistics, McNemar test, Fisher's exact test and analysis of variance models. Continuous variables were characterised using means and standard errors for normally distributed variables and median and 
Table 2 Characteristics of frail and non-frail patients

\begin{tabular}{lcc}
\hline & $\begin{array}{l}\text { Non-frail } \\
n=102\end{array}$ & $\begin{array}{l}\text { Frail } \\
n=49\end{array}$ \\
\hline Women, \% $(n)$ & $33(34)$ & $47(23)$ \\
Age (median, range), years & $71(60-93)$ & $74(61-96)$ \\
60-69, \% $(n)$ & $45.1(46)$ & $30.6(15)$ \\
$70-79, \%(n)$ & $36.3(37)$ & $38.8(19)$ \\
$\geq 80, \%(n)$ & $18.6(19)$ & $30.6(15)$ \\
Operated during the admission, \% $(n)$ & $44.1(45)$ & $28.6(14)$ \\
Charlson Comorbidity Index, estimated & $2.0(0.1)$ & $4.0(0.1)^{* *}$ \\
$\quad$ mean (SE) & & \\
Risk scores, estimated mean (SE) & & \\
Outpatient Bleeding Risk Index & $1.7(0.1)$ & $1.9(0.1)^{*}$ \\
$\quad($ OBRI) & $7.0(0.2)$ & $7.0(0.3)$ \\
Anticoagulation and risk factors & & \\
$\quad($ ATRIA) & $3.2(0.1)$ & $3.5(0.2)$ \\
HEMORR2HAGES & $3.0(0.1)$ & $3.4(0.2)$ \\
HAS-BLED & $1.1(0.1)$ & $1.3(0.1)$ \\
CHADS $_{2}$ & $2.0(0.1)$ & $2.5(0.2)^{*}$ \\
CHA $_{2}$ DS \\
\hline
\end{tabular}

An analysis of variance model including sex, age group, atrial fibrillation and frailty was utilised for comparing the difference between frailty groups

$S E$ standard error, $* p<0.05 ; * * p<0.001$ compared to non-frail category

interquartile range of values for variables that were not normally distributed. The normality of variables was evaluated visually and tested with the Shapiro-Wilk test. Square root transformation was used when necessary to make variables more normally distributed. The main statistical analyses for risk scores were performed using analysis of variance. Sex, age group, atrial fibrillation and frailty were included in all models.

The number of drugs used was primarily examined using repeated analysis of variance. Measurements from different time points for the same person were considered repeated measurements. Sex, age group, atrial fibrillation and frailty were included in all models. Furthermore, multiple interaction terms were included to compare subgroups sufficiently within the model.

The McNemar test was used to test statistical significance of the differences in the use of individual drugs between time points, and the Fisher's exact test was used to test statistical significance of the differences between frailty groups. More complicated models were not fitted to data on individual drugs because of the small sample size.

The statistical significance level was set at 0.05 in all tests (two-tailed). Analyses were performed using the SAS system, version 9.4 for Windows (SAS Institute Inc., Cary, NC, USA).

\section{Results}

We recruited 151 inpatients ( 94 were male and 57 were female), of whom 102 were non-frail and 49 frail. The characteristics, age distribution and risk scores are presented in Table 2. There was a slight preponderance of male individuals and patients in the younger age categories in the nonfrail group compared with the frail group ( $p=0.11$ and $p=$ 0.14 , respectively). The majority of calculated risk scores for bleeding and stroke were slightly higher in frail patients; yet a statistically significant difference was observed only for the Outpatient Bleeding Risk Index score (1.7 vs 1.9 for non-frail and frail patients, respectively, $p=0.0446$ ) and the $\mathrm{CHA}_{2} \mathrm{DS}_{2}$-VASc score (2.0 vs 2.5 for non-frail and frail patients, respectively, $p=0.0164)$. Instead, the Charlson Comorbidity Index was significantly higher for frail patients (4.0) compared with non-frail patients $(2.0, p<0.0001)$. No statistically significant differences were observed in the measured laboratory parameters (data not shown).

\subsection{Medication and Frailty}

Medication data before and after warfarin initiation were available for 98 non-frail and 47 frail patients. Frail patients were using on average 10.7 regular medications before warfarin initiation and 9.8 at discharge; in nonfrail patients, the corresponding numbers were 7.1 and 7.6 ( $p<0.0001$ and $p=0.0228$ between frail and non-frail groups, respectively) (Table 3). A sex-specific effect was observed when the number of regular medications before and after warfarin initiation were compared. Frail male patients had a higher number of regular medications than their non-frail counterparts before warfarin initiation $(p<$ 0.001 ), whereas in non-frail male patients, the number of medications increased significantly after warfarin initiation $(p=0.0010)$. In the non-frail group, female patients had more medications before warfarin initiation than male patients ( 8.6 vs. 5.7 , respectively, $p=0.068$ ). When both sexes were considered together, the within-group change was not significant in frail and non-frail patients.

Cardiovascular drugs was the most commonly used drug class both in non-frail and frail patients, used by over $80 \%$ of the patients (Table 4). The use of beta-blockers, particularly, showed a tendency for increased use in both patient groups after warfarin initiation. Systemic antimicrobials, including antibiotics and antifungals, were used especially by frail patients before warfarin initiation ( $14.9 \%$ vs. $5.1 \%$ in non-frail patients), but their use increased in both patient categories during the hospital stay so that almost $25 \%$ of patients were taking an antiinfective medication at discharge. 


\subsection{Interacting Drugs}

The use of drugs potentially interacting with warfarin was analysed by individual medication, medication class and by interaction mechanism (Table 5). Antithrombotics and analgesics were the most commonly used interacting medications both in non-frail and frail patients before warfarin initiation. Acetylsalicylic acid was used by half of all patients, whereas the use of clopidogrel was more common in frail patients $(27.1 \%)$ compared with non-frail patients $(9.9 \%, p=0.0135)$. An anticipated and statistically significant decrease was seen in the use of both clopidogrel and acetylsalicylic acid after warfarin initiation; approximately $10 \%$ of patients in both patient groups were using acetylsalicylic acid and only a few patients were taking clopidogrel at discharge. On the contrary, the use of enoxaparin significantly increased during hospital stay in both frailty categories $(p=0.0029$ and $p=$ 0.0196 , for non-frail and frail groups, respectively). The use of acetaminophen was nearly two-fold in frail patients compared with non-frail patients before warfarin initiation (63.8\% vs. $35.7 \%$, respectively, $p=0.0014)$. After warfarin initiation, the use of acetaminophen increased in non-frail patients and decreased in frail patients, with the change being statistically significant in the former group. At discharge, about half of the patients in both groups were using acetaminophen. Less than $10 \%$ of patients in both groups were using non-steroidal anti-inflammatory drugs (NSAIDs) before warfarin initiation and no users

Table 3 Mean number of drugs in use before (home) and after (discharge) warfarin initiation stratified by frailty, sex and presence of atrial fibrillation (AF)

\begin{tabular}{lcc}
\hline & Home mean (SE) & Discharge mean (SE) \\
\hline Non-frail, $n=98$ & $7.1(0.44)$ & $7.6(0.44)$ \\
AF & $8.4(0.59)$ & $8.6(0.59)$ \\
Non-AF & $5.8(0.67)$ & $6.7(0.68)$ \\
Male & $5.7(0.55)$ & $7.5(0.55)^{* * *}$ \\
Female & $8.6(0.72) \dagger$ & $7.8(0.73)$ \\
Frail, $n=47$ & $10.7(0.60)^{* *}$ & $9.8(0.60)^{*}$ \\
AF & $12.4(0.81)^{*}$ & $11.3(0.81)$ \\
Non-AF & $8.9(0.88)$ & $8.3(0.89)$ \\
Male & $11.6(0.82)^{* *}$ & $10.2(0.81)$ \\
Female & $9.7(0.87)$ & $9.4(0.89)$ \\
\hline
\end{tabular}

A repeated analysis of variance model was utilised for comparing the difference between frailty groups

$S E$ standard error, ${ }^{*} p<0.05$ compared to corresponding group of non-frail patients; $* * p<0.001$ compared to corresponding group of non-frail patients; $* * * p<0.05$ compared to number of drugs used prior to warfarin initiation (home) within the subgroup; $\nmid p<0.05$ compared to number of drugs used prior to warfarin initiation (home) in non-frail male patients was detected thereafter. An opposite trend was observed for tramadol, the use of which increased from $0.0 \%$ before warfarin initiation to $7.1 \%$ thereafter for non-frail patients and from $4.2 \%$ to $8.3 \%$ in frail patients, respectively.

The use of senna glycosides increased in both frail and non-frail patients during hospitalisation, being more common in frail patients (Table 5). The use of statins and fibrates was of a similar magnitude and remained as such in non-frail and frail patients over the hospital stay. The use of antidepressants remained low in both frailty groups.

The use of CYP2C9 inhibitors increased statistically significantly in both frailty groups. The increase was seen especially in non-frail patients and was mainly due to amiodarone; its contribution in the frail patients was smaller. No concomitant use of fluconazole (a CYP2C9 inhibitor) or CYP2C9 inducing drugs was observed; metronidazole was used at discharge by a single patient only in each group (data not shown). We also examined the use of known CYP3A4 inhibitors, which inhibit the metabolism of $R$-warfarin (Table 5). Fluoxetine was the only drug initiated over the admission, whereas the use of verapamil and diltiazem decreased over the admissions. No concomitant use was detected for other studied CYP3A4 inhibitors. When the proportions of interacting drug users at discharge were compared between frailty categories, no statistically significant differences were observed (Table 5).

\section{Discussion}

In this observational study, we examined the association between frailty and the exposure to potential interactions with warfarin in a real-care setting. We observed similar changes in the use of interacting drugs in both frail and nonfrail groups between admission and discharge except for acetaminophen and amiodarone. After warfarin initiation, we found no significant differences in warfarin drug interaction frequencies between frail and non-frail patients. Overall, the exposure to interacting drugs was modest in both groups and potentially serious drug interactions were few. A slight decrease in the total number of medications was observed between admission and discharge in frail patients, whereas the opposite was seen in non-frail patients.

The results of this study are encouraging as they suggest that concomitant medication as a risk factor for bleeding is taken into account when warfarin is initiated regardless of patients' frailty status. The study was conducted in a tertiary hospital and the patients included were predominantly treated in general medicine, cardiothoracic surgery, cardiology, and neurology units where warfarin is commonly prescribed and clinicians are likely to be well aware of warfarin drug interactions. The hospital setting may also have enabled the rationalising of medication in frail patients resulting in 
Table 4 Drug use before (home) and after (discharge) warfarin initiation in frailty categories expressed as the proportions (\%) and number of users (in brackets)

\begin{tabular}{|c|c|c|c|c|}
\hline & \multicolumn{2}{|c|}{ Non-frail, $n=98$} & \multicolumn{2}{|c|}{ Frail, $n=47$} \\
\hline & Home & Discharge & Home & Discharge \\
\hline GI drugs & $37.8(37)$ & $57.1(56)$ & $74.5(35)$ & $78.7(37)$ \\
\hline DM drugs & $21.4(21)$ & $20.4(20)$ & $36.2(17)$ & $34.0(16)$ \\
\hline Insulins & $5.1(5)$ & $7.1(7)$ & $21.3(10)$ & $19.1(9)$ \\
\hline Oral DM drugs & $19.4(19)$ & $16.3(16)$ & $27.7(13)$ & $25.5(12)$ \\
\hline Vitamins and minerals & $29.6(29)$ & $35.7(35)$ & $31.9(15)$ & $36.2(17)$ \\
\hline Antithrombotics & $57.1(56)$ & $27.6(27)$ & $68.1(32)$ & $27.7(13)$ \\
\hline Heparins & $3.1(3)$ & $16.3(16)$ & $4.3(2)$ & $19.1(9)$ \\
\hline Antiplatelets & $56.1(55)$ & $12.2(12)$ & $66.0(31)$ & $14.9(7)$ \\
\hline Acetylsalicylic acid & $50.0(49)$ & $11.2(11)$ & $48.9(23)$ & $8.5(4)$ \\
\hline Antianemic preparations & $6.1(6)$ & $8.2(8)$ & $19.1(9)$ & $14.9(7)$ \\
\hline Cardiovascular drugs & $78.6(77)$ & $88.8(84)$ & $91.5(43)$ & $93.6(44)$ \\
\hline Diuretics & $37.8(37)$ & $37.8(37)$ & $46.8(22)$ & $46.8(22)$ \\
\hline Beta blockers & $31.6(31)$ & $51.0(50)$ & $40.4(19)$ & $55.3(26)$ \\
\hline Calcium blockers & $30.6(30)$ & $22.4(22)$ & $36.2(17)$ & $23.4(11)$ \\
\hline RA system drugs & $62.2(61)$ & $58.2(57)$ & $66.0(31)$ & $55.3(26)$ \\
\hline Statins & $54.1(53)$ & $60.2(59)$ & $55.3(26)$ & $48.9(23)$ \\
\hline Urological drugs and sex hormones & $10.2(10)$ & $6.1(6)$ & $4.3(2)$ & $2.1(1)$ \\
\hline Hormonal preparations & $17.3(17)$ & $15.3(15)$ & $19.1(9)$ & $21.3(10)$ \\
\hline Corticosteroids & $7.1(7)$ & $7.1(7)$ & $8.5(4)$ & $10.6(5)$ \\
\hline Systemic antimicrobials & $5.1(5)$ & $24.5(24)$ & $14.9(7)$ & $25.5(12)$ \\
\hline Antineoplastics and immunomodulators & $4.1(4)$ & $4.1(4)$ & $10.6(5)$ & $10.6(5)$ \\
\hline Musculoskeletal system drugs & $24.5(24)$ & $12.2(12)$ & $21.3(10)$ & $10.6(5)$ \\
\hline NSAID $^{\mathrm{a}}$ & $9.2(9)$ & $0(0)$ & $6.4(3)$ & $0(0)$ \\
\hline Antigout drugs & $8.2(8)$ & $9.2(9)$ & $6.4(3)$ & $6.4(3)$ \\
\hline Bisphosphonates & $0(0)$ & $1.0(1)$ & $6.4(3)$ & $4.3(2)$ \\
\hline Nervous system & $52.0(51)$ & $64.3(63)$ & $80.9(38)$ & $70.2(33)$ \\
\hline Acetaminophen & $35.7(35)$ & $49.0(48)$ & $63.8(30)$ & $53.2(25)$ \\
\hline Opioids & $8.2(8)$ & $26.5(26)$ & $34.0(16)$ & $25.5(12)$ \\
\hline Antiepileptics & $6.1(6)$ & $7.1(7)$ & $6.4(3)$ & $8.5(4)$ \\
\hline Antipsychotics & $4.1(4)$ & $2.0(2)$ & $10.6(5)$ & $10.6(5)$ \\
\hline Antidepressants & $14.3(14)$ & $18.4(18)$ & $25.5(12)$ & $21.3(10)$ \\
\hline Non-selective MAO reuptake inhibitors & $3.1(3)$ & $3.1(3)$ & $8.5(4)$ & $4.3(2)$ \\
\hline SSRI & $5.1(5)$ & $8.2(8)$ & $2.1(1)$ & $2.1(1)$ \\
\hline MAO-A inhibitors & $0(0)$ & $0(0)$ & $4.3(2)$ & $2.1(1)$ \\
\hline Other antidepressants & $6.1(6)$ & $8.2(8)$ & $10.6(5)$ & $12.8(6)$ \\
\hline COPD and asthma drugs & $23.5(23)$ & $16.3(16)$ & $27.7(13)$ & $31.9(15)$ \\
\hline Ophthalmic drugs & $7.1(7)$ & $7.1(7)$ & $6.4(3)$ & $6.4(3)$ \\
\hline
\end{tabular}

$C O P D$ chronic obstructive pulmonary disease, $D M$ diabetes mellitus, $G I$ gastrointestinal, $M A O$ monoamine oxidase, $M A O-A$ monoamine oxidase-A, NSAID non-steroidal anti-inflammatory drug, RA system, renin-angiotensin system, SSRI selective serotonin reuptake inhibitor

${ }^{a}$ Excluding glucosamine

the decrease in the total number of drugs. On the contrary, the minor increase in total drug number seen in non-frail patients may be explained by better health status prior to admission, as indicated by the lower Charlson Comorbidity Index, and by the initiation of drug therapy to treat the cause of hospitalisation.
Of interacting drugs, the most notable changes were observed for antithrombotic medications. The significantly increased use of enoxaparin in both frailty groups may be explained by bridging the subtherapeutic period of warfarin therapy shortly after its initiation. The opposite was observed for antiplatelet drugs: in both groups, only approximately 
Table 5 Proportions of potentially interacting drug users prior (home) and after (discharge) warfarin initiation (home) by frailty

\begin{tabular}{|c|c|c|c|c|}
\hline \multirow[t]{2}{*}{$\%(n)$} & \multicolumn{2}{|c|}{ Non-frail, $n=98$} & \multicolumn{2}{|l|}{ Frail, $n=47$} \\
\hline & Home & Discharge & Home & Discharge \\
\hline Enoxaparin & $3.1(3)$ & $15.3(16)^{* *}$ & $4.3(2)$ & $19.1(9)^{*}$ \\
\hline Clopidogrel & $10.2(10)$ & $2.0(2)^{* *}$ & $27.7(13) * * *$ & $6.4(3)^{* *}$ \\
\hline Acetylsalisylic acid & $50.0(49)$ & $11.2(11)^{* *}$ & $48.9(23)$ & $8.5(4) * *$ \\
\hline NSAIDs $^{\mathrm{a}}$ & $9.2(9)$ & $0.0(0)^{* *}$ & $6.4(3)$ & $0.0(0)$ \\
\hline Acetaminophen & $35.7(35)$ & $49.0(48)^{*}$ & $63.8(30)^{* * *}$ & $53.2(25)$ \\
\hline Tramadol & $0.0(0)$ & $7.1(7)^{* *}$ & $4.3(2)$ & $8.5(4)$ \\
\hline Senna glycosides & $5.1(5)$ & $18.4(18)^{* *}$ & $12.8(6)$ & $29.8(14)^{* *}$ \\
\hline Simvastatin & $11.2(11)$ & $9.2(9)$ & $12.8(6)$ & $12.8(6)$ \\
\hline Rosuvastatin & $15.3(15)$ & $17.3(17)$ & $14.9(7)$ & $10.6(5)$ \\
\hline SSRIs $^{\mathrm{b}}$ & $5.1(5)$ & $8.2(8)$ & $2.1(1)$ & $2.1(1)$ \\
\hline SNRIs $^{c}$ & $4.1(4)$ & $4.1(4)$ & $6.4(3)$ & $8.5(4)$ \\
\hline Oral anti-infectives ${ }^{\mathrm{d}}$ & $0.0(0)$ & $5.1(5)^{*}$ & $2.1(1)$ & $6.4(3)$ \\
\hline CYP2C9 inhibitors ${ }^{\mathrm{e}}$ & $3.1(3)$ & $20.4(20)^{* *}$ & $10.6(5)$ & $19.1(9)^{*}$ \\
\hline Amiodarone & $0.0(0)$ & $16.3(16)^{* *}$ & $2.1(1)$ & $6.4(3)$ \\
\hline Fibrates & $3.1(3)$ & $2.0(2)$ & $6.4(3)$ & $6.4(3)$ \\
\hline CYP3A4 inhibitors & $12.2(12)$ & $11.2(11)$ & $10.6(5)$ & $6.4(3)$ \\
\hline Corticosteroids ${ }^{\mathrm{f}}$ & $6.1(6)$ & $7.1(7)$ & $8.5(4)$ & $8.5(4)$ \\
\hline Levothyroxine sodium & $10.2(10)$ & $10.2(10)$ & $10.6(5)$ & $10.6(5)$ \\
\hline Others $^{\mathrm{g}}$ & $4.1(4)$ & $3.1(3)$ & $6.4(3)$ & $6.4(3)$ \\
\hline
\end{tabular}

CYP cytochrome P450, NSAID non-steroidal anti-inflammatory drug, SNRI serotonin-norepinephrine reuptake inhibitor, SSRI selective serotonin reuptake inhibitor, ${ }^{*} p<0.05$ compared to the proportion of users at home within the frailty category; $* * p<0.01$ compared to the proportion of users at home within the frailty category; ${ }^{* *} p<0.05$ compared to the proportion of users at home in non-frail category

McNemar test was used for comparing the differences in the use of individual drugs between time points, and Fisher's exact test was for comparing the differences between frailty groups

No statistically significant differences were observed in the proportions of users between frailty categories at discharge

${ }^{a}$ NSAIDs include indomethacin, meloxicam and ibuprofen (combination products)

${ }^{\mathrm{b}}$ SSRIs include fluoxetine, citalopram, paroxetine, sertraline and escitalopram

${ }^{\mathrm{c}}$ SNRIs include venlafaxine and duloxetine

${ }^{\mathrm{d}}$ Oral anti-infectives include dicloxacillin, sulfamethoxazole and trimethoprim, moxifloxacin and metronidazole

${ }^{\text {e}} \mathrm{CYP} 2 \mathrm{C} 9$ inhibitors include amiodarone, fenofibrate, gemfibrozil, sulfamethoxazole-trimethoprim and metronidazole

${ }^{\mathrm{f}}$ Corticosteroids include dexamethasone, prednisolone, hydrocortisone and cortisone

${ }^{\mathrm{g}}$ Others include mesalazine, dipyridamole, carbimazole, mercaptopurine, azathioprine and phenytoin

every tenth patient continued on acetylsalicylic acid and few patients had clopidogrel in use at discharge. We presume that these patients who continued on antiplatelet drugs had an indication for dual antithrombotic medication as the indication for medications other than warfarin was not documented in the case notes. The more frequent use of clopidogrel prior to warfarin initiation in frail patients may be attributable to more common cardiovascular morbidity compared with non-frail patients. Furthermore, the vast decrease in the use of antiplatelet drugs may also result from the switch to warfarin because of clinical reasoning rather than avoiding the interaction.

The use of NSAIDs was modest before warfarin initiation in all patients and no use was detected in either of the frailty groups after warfarin initiation. In earlier studies,
NSAIDs have been among the most common interacting drugs in inpatients and outpatients taking warfarin $[3,4,9$, 27]. This trend seems to have changed and similar results to ours have been recently published in large register-based studies $[11,28,29]$. These findings may indicate increased awareness of clinicians of the increased bleeding risk associated with concomitant use of NSAIDs with warfarin. In our study population, the avoidance of prescribing NSAIDs may have contributed to the increased use of tramadol and acetaminophen in non-frail patients. Moreover, operations were more common in non-frail compared with frail patients $(44.1 \%$ vs $28.5 \%, p=0.06)$ and the analgesic effect of NSAIDs may have been considered inadequate for postoperative pain treatment. However, neither tramadol nor acetaminophen are trouble-free in patients taking warfarin. 
Tramadol impairs platelet function by inhibiting serotonin reuptake to platelets [30] and its concomitant use with warfarin is also associated with excess anticoagulation [31-34]. A metabolite of acetaminophen, $\mathrm{N}$-acetyl- $p$-benzoquinone, interferes with vitamin $\mathrm{K}$ metabolism and the synthesis of coagulation factors [35] thereby potentiating the warfarin response [36-38]. Moreover, acetaminophen may also have antiaggregatory effects [39]. Parallel results of increased use of tramadol and avoidance of NSAIDs were also reported by a Swedish register-based study, indicating that the warfarintramadol interaction may be poorly known among clinicians [28]. Altogether, pain management in patients receiving warfarin is a clinical challenge because of various types of drug interactions between commonly used analgesics and warfarin

Another noteworthy finding in our study was the increase in the use of CYP2C9 inhibiting drugs in both groups. In non-frail patients, amiodarone constituted $80 \%$ of the CYP2C9 inhibitor use at discharge whereas in frail patients amiodarone, fibrates and oral anti-infectives each comprised one third of these interactions. The common use of amiodarone in our study population is most likely explained by cardiac surgery procedures, e.g. heart valve replacement and cardiac bypass surgery, performed in tertiary hospitals, and these would have been more likely in non-frail patients. Although the warfarin-amiodarone interaction is of clinical significance and leads to an increased anticoagulant effect by inhibiting warfarin metabolism [40], long-term use and stable dosing of amiodarone enables the control of the effect of interaction by adjusting warfarin dose according to INR values. The same principle applies also to CYP2C9 inhibiting fibrates whereas oral anti-infectives are more challenging: not only do they inhibit the metabolism of warfarin but they also affect the gut microbiota disturbing the vitamin K production. Therefore, the effects of anti-infectives on warfarin response are difficult to predict and control even after the course. Nevertheless, the number of interactions caused by oral anti-infectives was few and by CYP2C9 inhibitors modest in total, mainly comprising amiodarone, the effect of which (as discussed) is possible to compensate by warfarin dosing.

We also detected a significant increase in senna use in both non-frail and frail patients after warfarin initiation. Constipation is a common problem in hospitalised elderly patients [41]. Senna increases bowel motility thereby potentially decreasing the absorption of vitamin $\mathrm{K}$ and resulting in elevated INR values [42]. In patients newly started on warfarin, senna use may lead to exaggerated anticoagulation and early bleeding complications despite close INR monitoring during the hospital admission. The use of simvastatin and rosuvastatin remained at a similar order of magnitude in both frailty groups before and after warfarin initiation. The clinical significance of the warfarin-statin interaction has been a matter of discrepancy in previous literature [43, 44]. A recent nationwide database study found no association between the concomitant use of statins and an increased rate of bleeding in new warfarin users [45]. We suspect that the inconsistency in the literature about the significance of the warfarin-statin interaction as well as the clinical experience of prescribing physicians on the concomitant use may explain the stable use of statins in our study.

This study has some important strengths and limitations. The main strength of our study is the comprehensive and detailed data describing real-world drug use and capturing an incident cohort of warfarin users without previous oral anticoagulation use. As drugs were administered during the inpatient care, the actual exposure to interacting medication is highly likely. The most significant limitation of this study is the small sample size. The insignificant differences in the interaction drug use between frailty classes may be due to the small number of observations. This study was performed at a single hospital and the results may be influenced by local practices, which may hinder the generalisation of our results.

\section{Conclusions}

Our study showed that frailty status is not associated with exposure to drug interactions increasing the bleeding risk in new warfarin users. Acetaminophen, CYP2C9 inhibitors and senna were the most common interacting drugs in all patients. Overall, the initiation of warfarin therapy appeared to be well considered in relation to other medications independent of a patient's frailty status. However, future studies including larger study populations and longer follow-up are required to confirm our findings and to study the sustainability of medication changes.

Acknowledgements Open access funding was provided by the University of Turku (UTU) including Turku University Central Hospital. We thank Melinda Stanners, PhD for her effort in patient recruitment and data collection. We also thank the clinical pharmacists and other personnel of Royal Adelaide Hospital who helped identify eligible patients and invited them to participate. Mrs. Todorka Grammatopoulos is acknowledged for her valuable assistance in patient file management.

\section{Compliance with Ethical Standards}

Funding This study was supported by a grant from the Hospital District of South-Western Finland.

Conflict of interest Milka Hauta-aho, Simo Teperi, Maarit J. Korhonen, Nicholas Farinola, Sally Johns, Sepehr Shakib and Risto Huupponen have no conflicts of interest that are directly relevant to this content of this article. J. Simon Bell is supported by a National Health 
and Medical Research Council Dementia Research Leadership Fellowship.

Ethical approval This study was approved by the institutional human research ethics committee (Royal Adelaide Hospital Protocol No. 120911).

Consent to participate A written informed consent was obtained from all participants prior to inclusion. For patients with cognitive impairment, a third-party consent was obtained from patients' next of kin, carer or significant other.

Open Access This article is licensed under a Creative Commons Attribution-NonCommercial 4.0 International License, which permits any non-commercial use, sharing, adaptation, distribution and reproduction in any medium or format, as long as you give appropriate credit to the original author(s) and the source, provide a link to the Creative Commons licence, and indicate if changes were made. The images or other third party material in this article are included in the article's Creative Commons licence, unless indicated otherwise in a credit line to the material. If material is not included in the article's Creative Commons licence and your intended use is not permitted by statutory regulation or exceeds the permitted use, you will need to obtain permission directly from the copyright holder.To view a copy of this licence, visit http://creativecommons.org/licenses/by-nc/4.0/.

\section{References}

1. Fang MC, Go AS, Hylek EM, Chang Y, Henault LE, Jensvold $\mathrm{NG}$, et al. Age and the risk of warfarin-associated hemorrhage: the anticoagulation and risk factors in atrial fibrillation study. $\mathrm{J}$ Am Geriatr Soc. 2006;54:1231-6.

2. Hylek EM, Evans-Molina C, Shea C, Henault LE, Regan S. Major hemorrhage and tolerability of warfarin in the first year of therapy among elderly patients with atrial fibrillation. Circulation. 2007;115:2689-96.

3. Hauta-Aho M, Tirkkonen T, Vahlberg T, Laine K. The effect of drug interactions on bleeding risk associated with warfarin therapy in hospitalized patients. Ann Med. 2009;41:619-28.

4. Vitry AI, Roughead EE, Ramsay EN, et al. Major bleeding risk associated with warfarin and co-medications in the elderly population. Pharmacoepidemiol Drug Saf. 2011;20:1057-63.

5. Perera V, Bajorek BV, Matthews S, Hilmer SN. The impact of frailty on the utilisation of antithrombotic therapy in older patients with atrial fibrillation. Age Ageing. 2009;38:156-62.

6. Lefebvre M-CD, St-Onge M, Glazer-Cavanagh M, Bell L, Kha Nguyen JN, Viet-Quoc Nguyen P, et al. The effect of bleeding risk and frailty status on anticoagulation patterns in octogenarians with atrial fibrillation: the FRAIL-AF study. Can J Cardiol. 2016;32:169-76.

7. Gnjidic D, Hilmer SN, Blyth FM, Naganathan V, Cumming RG, Handelsman DJ, et al. High-risk prescribing and incidence of frailty among older community-dwelling men. Clin Pharmacol Ther. 2012;91:521-8.

8. Cullinan S, O'Mahony D, O'Sullivan D, Byrne S. Use of a frailty index to identify potentially inappropriate prescribing and adverse drug reaction risks in older patients. Age Ageing. 2016;45:115-20.

9. Gavronski M, Hartikainen S, Zharkovsky A. Analysis of potential interactions between warfarin and prescriptions in Estonian outpatients aged 50 years or more. Pharm Pract (Granada). 2012;10:9-16.
10. Suh D-C, Nelson WW, Choi JC, Choi I. Risk of hemorrhage and treatment costs associated with warfarin drug interactions in patients with atrial fibrillation. Clin Ther. 2012;34:1569-82.

11. Rikala M, Hauta-Aho M, Helin-Salmivaara A, Lassila R, Korhonen MJ, Huupponen R. Co-prescribing of potentially interacting drugs during warfarin therapy: a population-based register study. Basic Clin Pharmacol Toxicol. 2015;117:126-32.

12. Rikala M, Kastarinen H, Tiittanen P, Huupponen R, Korhonen MJ. Natural history of bleeding and characteristics of early bleeders among warfarin initiators: a cohort study in Finland. Clin Epidemiol. 2016;8:23-35.

13. Hilmer SN, Perera V, Mitchell S, Murnion BP, Dent J, Bajorek $\mathrm{B}$, et al. The assessment of frailty in older people in acute care. Aust J Ageing. 2009;28:182-8.

14. Bennett A, Gnjidic D, Gillett M, Carroll P, Matthews S, Johnell $\mathrm{K}$, et al. Prevalence and impact of fall-risk-increasing drugs, polypharmacy, and drug-drug interactions in robust versus frail hospitalised falls patients: a prospective cohort study. Drugs Aging. 2014;31:225-32.

15. Thai M, Hilmer S, Pearson S-A, Reeve E, Gnjidic D. Prevalence of potential and clinically relevant statin-drug interactions in frail and robust older inpatients. Drugs Aging. 2015;32:849-56.

16. Rolfson DB, Majumdar SR, Tsuyuki RT, Tahir A, Rockwood K. Validity and reliability of the Edmonton Frail Scale. Age Ageing. 2006;35:526-9.

17. Böttiger Y, Laine K, Andersson ML, Korhonen T, Molin B, Ovesjö M-L, et al. SFINX: a drug-drug interaction database designed for clinical decision support systems. Eur J Clin Pharmacol. 2009;65:627-33.

18. Charlson ME, Pompei P, Ales KL, MacKenzie CR. A new method of classifying prognostic comorbidity in longitudinal studies: development and validation. J Chronic Dis. 1987;40:373-83.

19. Olesen JB, Lip GYH, Hansen PR, Lindhardsen J, Ahlehoff O, Andersson C, et al. Bleeding risk in "real world" patients with atrial fibrillation: comparison of two established bleeding prediction schemes in a nationwide cohort. J Thromb Haemost. 2011;9:1460-7.

20. Beyth RJ, Quinn LM, Landefeld CS. Prospective evaluation of an index for predicting the risk of major bleeding in outpatients treated with warfarin. Am J Med. 1998;105:91-9.

21. Fang MC, Go AS, Chang Y, Borowsky LH, Pomernacki NK, Udaltsova N, et al. A new risk scheme to predict warfarin-associated hemorrhage. J Am Coll Cardiol. 2011;58:395-401.

22. Gage BF, Yan Y, Milligan PE, Waterman AD, Culverhouse R, Rich MW, et al. Clinical classification schemes for predicting hemorrhage: results from the National Registry of Atrial Fibrillation (NRAF). Am Heart J. 2006;151:713-9.

23. Pisters R, Lane DA, Nieuwlaat R, de Vos CB, Crijns HJGM, Lip GYH. A novel user-friendly score (HAS-BLED) to assess 1-year risk of major bleeding in patients with atrial fibrillation. Chest. 2010;138:1093-100.

24. Lip GYH, Frison L, Halperin JL, Lane DA. Comparative validation of a novel risk score for predicting bleeding risk in anticoagulated patients with atrial fibrillation: the HAS-BLED (Hypertension, Abnormal Renal/Liver Function, Stroke, Bleeding History or Predisposition, Labile INR, Elderly, Drugs/Alcohol Concomitantly) score. J Am Coll Cardiol. 2011;57:173-80.

25. Gage BF, Waterman AD, Shannon W, Boechler M, Rich MW, Radford MJ. Validation of clinical classification schemes for predicting stroke: results from the National Registry of Atrial Fibrillation. JAMA. 2001;285:2864-70.

26. Lip GYH, Nieuwlaat R, Pisters R, Lane DA, Crijns HJGM. Refining clinical risk stratification for predicting stroke and thromboembolism in atrial fibrillation using a novel risk factor-based approach. Chest. 2010;137:263-72. 
27. Tragni E, Casula M, Pieri V, Favato G, Marcobelli A, Trotta MG, et al. Prevalence of the prescription of potentially interacting drugs. PLoS One. 2013;8:e78827.

28. Lindh JD, Andersson ML, Mannheimer B. Adherence to guidelines for avoiding drug interactions associated with warfarin: a nationwide Swedish Register study. PLoS One. 2014;9:e97388.

29. Ilomäki J, Helin-Salmivaara A, Huupponen R, Rikala M, Kirkpatrick CM, Korhonen MJ. Analgesic use before and after oral anticoagulant initiation: a population-based study in Finland. Eur J Clin Pharmacol. 2015;71:723-32.

30. Klotz U. Tramadol: the impact of its pharmacokinetic and pharmacodynamic properties on the clinical management of pain. Arzneimittelforschung. 2003;53:681-7.

31. Scher ML, Huntington NH, Vitillo JA. Potential interaction between tramadol and warfarin. Ann Pharmacother. 1997;31:646-7.

32. Sabbe JR, Sims PJ, Sims MH. Tramadol-warfarin interaction. Pharmacotherapy. 1998;18:871-3.

33. Juel J, Pedersen TB, Langfrits CS, Jensen SE. Administration of tramadol or ibuprofen increases the INR level in patients on warfarin. Eur J Clin Pharmacol. 2013;69:291-2.

34. Pottegård A, Meegaard PM, Holck LH, Christensen Rd, Madsen $\mathrm{H}$, Hallas J. Concurrent use of tramadol and oral vitamin $\mathrm{K}$ antagonists and the risk of excessive anticoagulation: a register-based nested case-control study. Eur J Clin Pharmacol. 2013;69:641-6.

35. Thijssen HH, Soute BA, Vervoort LM, Claessens JG. Paracetamol (acetaminophen) warfarin interaction: NAPQI, the toxic metabolite of paracetamol, is an inhibitor of enzymes in the vitamin $\mathrm{K}$ cycle. Thromb Haemost. 2004;92:797-802.

36. Mahé I, Bertrand N, Drouet L, Bal Dit Sollier C, Simoneau G, Mazoyer E, et al. Interaction between paracetamol and warfarin in patients: a double-blind, placebo-controlled, randomized study. Haematologica. 2006;91:1621-7.
37. Parra D, Beckey NP, Stevens GR. The effect of acetaminophen on the international normalized ratio in patients stabilized on warfarin therapy. Pharmacotherapy. 2007;27:675-83.

38. Zhang Q, Bal-dit-Sollier C, Drouet L, et al. Interaction between acetaminophen and warfarin in adults receiving long-term oral anticoagulants: a randomized controlled trial. Eur J Clin Pharmacol. 2011;67:309-14.

39. Munsterhjelm E, Munsterhjelm NM, Niemi TT, Ylikorkala O, Neuvonen PJ, Rosenberg PH. Dose-dependent inhibition of platelet function by acetaminophen in healthy volunteers. Anesthesiology. 2005; 103:712-7.

40. Heimark LD, Wienkers L, Kunze K, Gibaldi M, Eddy AC, Trager $\mathrm{WF}$, et al. The mechanism of the interaction between amiodarone and warfarin in humans. Clin Pharmacol Ther. 1992;51:398-407.

41. Noiesen E, Trosborg I, Bager L, Herning M, Lyngby C, Konradsen $\mathrm{H}$. Constipation: prevalence and incidence among medical patients acutely admitted to hospital with a medical condition. J Clin Nurs. 2014;23:2295-302.

42. Kittisupamongkol W, Nilaratanakul V, Kulwichit W. Nearfatal bleeding, senna, and the opposite of lettuce. Lancet. 2008;371:784

43. Schelleman H, Bilker WB, Brensinger CM, Wan F, Yang Y-X, Hennessy S. Fibrate/statin initiation in warfarin users and gastrointestinal bleeding risk. Am J Med. 2010;123:151-7.

44. Douketis JD, Melo M, Bell CM, Mamdani MM. Does statin therapy decrease the risk for bleeding in patients who are receiving warfarin? Am J Med. 2007;120(369):e9-14.

45. Korhonen MJ, Tiittanen P, Kastarinen H, Helin-Salmivaara A, Hauta-aho M, Rikala M, et al. Statins do not increase the rate of bleeding among warfarin users. Basic Clin Pharmacol Toxicol. 2018;123:195-201.

\section{Affiliations}

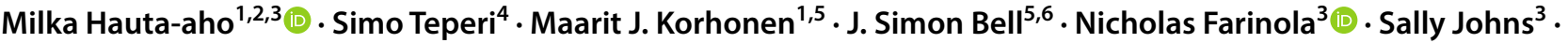 Sepehr Shakib ${ }^{3,7}$ (1) Risto Huupponen ${ }^{1,2}$}

1 Institute of Biomedicine, Research Centre for Integrative Physiology and Pharmacology, University of Turku, Kiinamyllynkatu 10, 20014 Turku, Finland

2 Clinical Pharmacology Unit, Turku University Hospital, Turku, Finland

3 Department of Clinical Pharmacology, Royal Adelaide Hospital, Adelaide, SA, Australia

4 Department of Biostatistics, University of Turku, Turku, Finland
5 Faculty of Pharmacy and Pharmaceutical Sciences, Centre for Medicine Use and Safety, Monash University, Melbourne, VIC, Australia

6 NHMRC Centre of Research Excellence in Frailty and Healthy Ageing, Adelaide, SA, Australia

7 Department of Clinical Pharmacology, Faculty of Health Science, University of Adelaide, Adelaide, SA, Australia 